\title{
Ausbildungsmodelle für Lehrpersonen der Sekundarstufe I in der Deutschschweiz \\ - Grundlinien und Konfliktlinien
}

\section{Andreas Hoffmann-Ocon}

Gestützt auf Archivalien der EDK befasst sich der Beitrag mit den Reformversuchen der Lehrpersonenausbildung für die Sekundarstufe I bzw. Volksschuloberstufe in der zweiten Hälfte des 20. Jahrhunderts in der deutschsprachigen Schweiz. Die Themen Akademisierung, universitäre Verortung und Schulstufenorientierung bildeten eine Konfliktlinie, welche die kantonalen an Schultypen angelehnten tradierten Ausbildungsstrukturen irritierte. In den bildungshistorischen Rekonstruktionen zeigt sich, dass sowohl die Reforminitianten als auch die Reformkritiker bemüht waren, ihre Positionen mit wissenschaftlicher Expertise zu untermauern. Vor dem Hintergrund einer Dokumentenanalyse gelangt der Beitrag zur Einschätzung, dass wissenschaftliche Bezugnahme allein nicht ausreichte, um einen Systemwechsel in der Lehrpersonenausbildung herbeizuführen.

Vor etwa 30 Jahren charakterisierte Lutz Oertel, wissenschaftlicher Mitarbeiter der Pädagogischen Abteilung der Erziehungsdirektion Kanton Zürich, in einem Artikel über die Probleme der Oberstufenreform die Geschichte der Zürcher Volksschule als eine Geschichte ungelöster Probleme der Volksschuloberstufe (Oertel, 1981). In Anlehnung an diese Interpretation könnte die jüngere Geschichte der Ausbildungs- und Studiengänge für Lehrpersonen der Sekundarstufe I (Sek I) ebenfalls als eine Geschichte ungelöster Probleme entworfen werden. So wie die 25 Kantone und Halbkantone seit 1848 ihr je eigenes Schulwesen entwickelten und pflegten, so vielgestaltig war die Volksschullehrerbildung. Das Spektrum von Ausbildungs- und Studiengängen reichte in der Regel in der deutschsprachigen Schweiz vom vierjährigen Seminar, das an die neunjährige Volksschule anschloss, bis zum sechssemestrigen Studiengang an der Universität mit einem späteren kantonalen Examen. In den meisten Kantonen bildete das Lehrerseminar Lehrpersonen für die Unter-, Mittel und Oberstufe der Volksschule aus, und die Lehrpersonen der Volksschuloberstufe, der Sek I, durchliefen je nach Schulzweig und -fächern an den entsprechenden Ausbildungsinstitutionen bzw. an der Universität eine Art zweite Ausbildungsphase (Bucher, 1970; Frey, 1969). Der Versuch, die Entwicklung von Ausbil- 
dungsmodellen für Lehrpersonen der Sekundarstufe I in der Deutschschweiz zu rekonstruieren, zu kontextualisieren und aus der Optik einer durch die unausweichliche Standortgebundenheit geprägten «Post-Bologna-Epoche» bildungshistorisch zu interpretieren, steht vor einigen Herausforderungen:

- Semantische Herausforderung: Da lange Zeit fraglich war, ob überhaupt eine Sek I in der deutschsprachigen Schweiz existierte, gab es Unklarheiten hinsichtlich der Begriffsbestimmung. Heinz Badertschers Definition zu Beginn der 1980er-Jahre zielte vor dem Hintergrund, dass eine Sek I in schweizerischer Sicht nicht existierte, «auf die letzte Phase der obligatorischen Schulzeit mit allen ihren unterschiedlichen Schultypen» (Badertscher, 1983, S. 21). Charakteristisch für diese Phase sollte ihre Zugehörigkeit zur Volksschule sein. Die Vielfalt der Schultypen auf dieser Stufe korrespondierte mit der Vielfalt an Ausbildungsgängen für Lehrpersonen.

- Kantonale Bildungspolitik als Herausforderung: Aus der Optik einer Sek I bedeutete bis vor etwa zehn Jahren die fehlende interkantonale Koordination, aber auch die starke Orientierung an Schultypen, dass Lehrpersonen für Schülerinnen und Schüler der gleichen Altersstufe «entweder keine spezifische Ausbildung oder einen besonderen Lehrgang» bis sechs Semester an einer Universität besuchten (Badertscher, 1983, S. 22). Was für die schweizerische Bildungspolitik insgesamt galt, galt vor allem auch für die Steuerung der Ausbildungsgänge für Lehrpersonen der Sek I: Mochten sich die Erziehungsdirektoren um die Abstimmung ihrer Konzeptionen auf kantonaler Ratsebene bemühen, so wenig waren sie mit ihrer Entscheidungskompetenz an die Verlautbarungen der EDK im eigenen Kanton gebunden (Rosenmund, 2011).

- Faktizität des Lehrpersoneneinsatzes als Herausforderung: Aus der (verschriftlichten) Intention kantonaler Entwürfe der Bildungspolitik, Lehrpersonen schultypen- oder zielstufengerecht auszubilden und einzusetzen, darf nicht voreilig auf die tatsächliche Praxis geschlossen werden. So konstatierte diesbezüglich die COHEP 2009, «dass in der Praxis seit einigen Jahren auf der Sek I ein Mangel an qualifizierten Lehrpersonen zu verzeichnen ist und deshalb Lehrpersonen der Primarstufe (...) angestellt wurden» (COHEP, 2009, S. 1). Die angesprochenen Lehrpersonen waren jedoch nicht für die Sek I ausgebildet. Dieser Umstand zog zunächst das Angebot von kantonalen Ergänzungsstudien nach sich, die den Lehrpersonen ein minimales Rüstzeug für den Unterricht auf der Zielstufe liefern und als Bedingung für eine Festanstellung dienen sollten. In den letzten Jahren etablierten einige pädagogische Hochschulen neben der Ausbildung für erfahrene Berufsleute ("Quereinsteiger») Zweit- bzw. konsekutive Masterstudiengänge für Personen mit Lehrdiplom für die Vorschulstufe / Primarstufe, die noch ein Diplom für die Sek I erwerben wollen. 
- Verschiedene Anspruchsgruppen als Herausforderung: Die Sek I befand sich stets - metaphorisch gesprochen - «im Wegkreuz von Volksbildung, Berufsausbildung und Gymnasialbildung» (Jenzer, 1998, S. 61). Dies führte zur Herausbildung unterschiedlicher Oberstufentypen, woraus Durchlässigkeitsprobleme entstanden.

- Herausforderung des trügerischen «Bologna-Scheins»: Die Eckwerte der heutigen Studiengänge für Lehrpersonen der Sek I wurden in einer Entwurfsphase der frühen 1990er-Jahre bestimmt, als von einer Bologna-Förmigkeit noch gar keine Rede war; die Umsetzungsphase der Studiengänge an pädagogischen Hochschulen nach dem Jahr 2000 war davon geprägt, ein Passungsverhältnis zwischen EDK-Reglement-konformen Studiengängen und Bologna-Anforderungen nachträglich herzustellen.

Allein diese Auswahl an begriffsdefinitorischen und bildungshistorischen Herausforderungen, die es in diesem Beitrag mit zu bedenken gilt, lassen eine Selbstbescheidung im Anspruch ratsam erscheinen. Einige Teilaspekte zu den Ausbildungsmodellen werden unberücksichtigt bleiben; so verfügen z.B. einige Kantone über Langzeitgymnasien bzw. Untergymnasien, die zwar zur Sek I gerechnet werden müssen, aber nicht zur Volksschuloberstufe gehören, wenn damit der Anschluss an die Primarstufe als Abschluss der Volksschulbildung gemeint ist. Die Ausbildung von Gymnasiallehrpersonen müssten bei einer umfangreichen Bearbeitung dieses Themas aufgegriffen werden.

Die Abkehr von der schultypenorientierten und die Hinwendung zur schulstufenorientierten Lehrpersonenausbildung für die Sek I wird oftmals mit den Studiengängen der pädagogischen Hochschulen in Verbindung gebracht. Diese Auffassung möchte ich folgend um eine bildungshistorische Perspektive erweitern, die Entwürfe einer stufenorientierten Lehrpersonenausbildung aus den frühen 1980er-Jahren als prägnante Vorformulierungen heutiger Ausbildungsmodelle versteht. In dem Beitrag wird aufgezeigt, dass ein von der EDK beauftragter Unterausschuss zur Reform der Lehrpersonenausbildung Massstäbe für aktuelle Sek-I-Studiengänge setzte, dabei jedoch die bis dahin tradierten Grundlinien der Ausbildungsmuster für Lehrerinnen und Lehrer der Volksschuloberstufe verliess und somit Konflikte mit Bildungsverwaltungen und Verbänden auslöste. Im Ergebnis möchte ich mit der vorliegenden Studie die These plausibilisieren, dass der bedeutsame Schlussbericht der Arbeitsgruppe Sekundarstufe I (AGS I) von 1983 der historisch vorzeitige Versuch war, die «Systemgrenzen» der altbewährten Strukturen von Sek-I-Ausbildungsmodellen zu überschreiten.

$\mathrm{Zu}$ Beginn werde ich die Grundlinien der Ausbildungsmodelle für Reallehrpersonen und Sekundarlehrpersonen seit den 1950er-Jahren bis zur Mitte der 1990er-Jahre skizzieren. Darauf folgend rekonstruiere ich auf der Basis von bisher unveröffentlichten Archivalien und Dokumenten der AGS I, die im Staatsarchiv Luzern $(\mathrm{StA} \mathrm{LU})^{1}$ verwahrt werden, zwei Beispiele von bildungs- 
politischen Konfliktlinien zur Sek-I-Ausbildung, die bis heute auf Argumentarien im Diskurs der Lehrerinnen- und Lehrerbildung in der deutschsprachigen Schweiz einwirken: Zum einen den Konflikt über die Akademisierung von Teilen der Ausbildung für Lehrpersonen der Volksschuloberstufe und zum anderen den Konflikt über die Orientierung der Ausbildungen an Schultypen oder Schulstufen. Insbesondere die Interpretation der Rekonstruktion der zweiten Konfliktlinie verweist auf das aktuelle Passungsproblem von stufenorientierter Lehrpersonenausbildung und real vorfindbarer schultypenorientierter Organisation der Sekundarstufe I in der deutschsprachigen Schweiz. Zu dem Einfluss tradierter Ausbildungsüberzeugungen auf aktuelle Studiengänge für Lehrpersonen der Sek I wird ein Deutungsangebot entworfen.

Die Rekonstruktions- und Interpretationsarbeit basiert auf der Implikation eines heteronomen bildungspolitischen Feldes, in dem vor allem Akteure aus der Wissenschaft, aus Bildungsverwaltungen und aus Standesvertretungen das Kräfteverhältnis zugunsten ihrer Optik zu verändern versuchten. Dabei konkurrenzierten sich Befürwortende einer neuen Ausbildungsstruktur mit den Verteidigenden der etablierten Ordnung im Zuge der Meinungsproduktion um den Entwurf eines "common sense» der angemessenen Lehrpersonenausbildung für die Sek I (Bourdieu, 2001, S. 12 ff.). Bedeutsam wird die Unterscheidung zwischen «reinem wissenschaftlichen Kapital», welches in hochselektiven wissenschaftlichen Organen Platz findet, und «institutionalisiertem wissenschaftlichen Kapital», das tendenziell als "Professionswissen» in nichtforschenden Einrichtungen der Lehrerinnen- und Lehrerbildung vor Gründung der pädagogischen Hochschulen distribuiert wurde (Bourdieu, 1998, S. 32).

Der archivalische Quellenkorpus, u.a. aus vielen Vernehmlassungsbemerkungen bestehend, bildet ein Archiv der Argumentarien, die möglicherweise nicht beliebig erweiterbar, sondern begrenzt sind. Zwar gewährt dieser Materialzugang eine quellen- und dokumentennahe Aufbereitung der Konfliktlinien, aber aus der Retrospektive von etwa 30 Jahren ist es auch schwierig einzuschätzen, welche Geltungsmacht und bildungspolitische Wirksamkeit den Dokumenten selbst zukam; hatte z.B. eine sehr langfädige Rückmeldung des Erziehungsdepartements eines Nichthochschulkantons eine grössere Wirkung als die knappen Worte der Bildungsdirektion eines Hochschulkantons? Wie einflussreich war z.B. eine kantonale Realschullehrerkonferenz im Verhältnis zu der «Konferenz von Direktoren der Sekundarlehrerausbildung in der deutschsprachigen Schweiz (KDS)» im Vernehmlassungsprozess? Die Bildung von Kontrastgruppen (nach bevölkerungsreichen und -armen Kantonen, nach reformierten, katholischen, gemischtkonfessionellen Kantonen, nach Kantonen mit und Kantonen ohne Lehrerausbildungsinstitutionen etc.) war durch die Auswahl der Vernehmlassungsrückmeldungen nicht möglich, da nicht alle Bildungsdirektionen und Verbände als Vernehmlassungspartner gleichermassen aussagekräftige Rückmeldungen geliefert haben. 


\section{Grundlinien der Entwicklung von Ausbildungsmo- dellen (1950er- bis 1990er-Jahre)}

\section{Ausbildungsmodelle für Reallehrpersonen}

Bis Ende der 1950er-Jahre lag in der deutschsprachigen Schweiz für Lehrpersonen, die in der Volksschuloberstufe mit Grundanforderungen arbeiteten, kein spezifisches Ausbildungsprogramm vor. In vielen Kantonen, Basel-Stadt bildete eine Ausnahme, umfasste die Primarschule nach herrschendem Verständnis sieben, acht oder neun Jahre des Schulobligatoriums (Archiv für das schweizerische Unterrichtswesen, 1965/66). Dieser Logik folgend unterrichteten in diesem Leistungszweig (Schulen ohne Mittelschulanschluss) Lehrpersonen mit Primarlehrerpatent. Die Situierung der Lehrpersonen für die Volksschuloberstufe wurde in der Regel dergestalt begründet, dass die dezentralen Schulstrukturen für diesen Leistungszweig eine "Allrounder-Reallehrkraft» erforderten, die ihr Augenmerk auf den "pädagogischen Bezug» zu den Schülerinnen und Schülern legt und Beziehungspflege zu den lokalen Berufslehren betreibt. Um diese Aufgaben erfüllen zu können, so lautete die generelle Annahme, reiche eine Ausbildung zur Primarlehrperson und allenfalls eine Zusatzausbildung aus, die auch einen Weiterbildungscharakter aufweisen könne (EDK, 1979; Pädagogische Arbeitsstelle der Erziehungs- und Kulturdirektion (PAEK), 1993).

Ein Modell spezifisch für Lehrpersonen in diesem Leistungszweig entwickelte erstmals 1959 der Kanton Zürich mit der an das Primarlehrerpatent anknüpfenden viersemestrigen Ausbildung am Real- und Oberschullehrerseminar. Diese Ausbildung zeichnete sich dadurch aus, dass neben dem Studium von Unterrichtsfächern bzw. Fachbereichen sowie deren Methodik bzw. speziellen Didaktik auch Studienanteile in einem erziehungswissenschaftlichen «Fachbereich» zu absolvieren waren (EDK, 1979, S. 3).

$\mathrm{Zu}$ Beginn der 1970er-Jahre führten die Kantone Aargau und Solothurn je eine einsemestrige Vollzeitausbildung für Oberstufenlehrer ein. Im Aargau wurde der Ausbildungsgang für die "Primaroberschule» an der Lehramtsschule am Sitz der Höheren Technischen Lehranstalt (HTL) in Brugg-Windisch zunächst nicht obligatorisch verlangt, d.h. er war fakultativ. Dagegen war im Kanton Solothurn für die Erlangung der Wahlfähigkeit an Primaroberschulen der Besuch des einjährigen Kurses obligatorisch. Beide kantonalen Ausbildungsgänge sahen verpflichtende Studien in pädagogischen, psychologischen und allgemeindidaktischen Lernbereichen vor (EDK, 1979). Wohl mit Orientierung an der Ausbildungskonzeption in Zürich richtete St. Gallen an seiner Sekundarlehramtsschule 1976 einen zweisemestrigen Kurs für Primarschullehrpersonen ein, die sich zu «Abschlussklassen»-Lehrpersonen ausbilden lassen wollten. Auch für diesen Kurs war typisch, dass er u.a. das Studium der «Pädagogik und Pädagogischen Psychologie» vorsah (EDK, 1979, S. 7). Ebenfalls ein "grundständiges» Studium stellte die dreisemestrige Ausbildung für Lehrer an der Sekundarschule (= Oberstufe der Primarschule) in Basel-Stadt und Basel-Land dar, die 1978 
aufgegleist am kantonalen Lehrerseminar Liestal BL durchgeführt wurde und ebenfalls neben dem Studium der Unterrichtsfächer und ihren Didaktiken Studienbereiche in Pädagogik vorsah (EDK, 1979, S. 13). Andere Kantone in der deutschsprachigen Schweiz, wie z.B. Schaffhausen, Thurgau und Appenzell $A R$, verlangten den Besuch von mehrwöchigen Ausbildungskursen.

\section{Ausbildungsmodelle für Sekundarlehrpersonen}

Die Tabelle 1 gibt darüber Auskunft, dass bereits in den 1960er-Jahren die Ausbildung zur Sekundarlehrperson einen dezidiert akademischen Anspruch hatte, der aber wegen der Kürze und der Fächervielfalt des Studiums im Vergleich zu anderen universitären Studiengängen nicht widerspruchsfrei war. Die meisten Kantone verpflichteten die Lehramtskandidaten zu einem vier- bis sechssemestrigen Studium an einer Universität oder an einer Lehramtsschule, die wiederum Studienmöglichkeiten in Kooperation mit einer Universität vorsah. In der Regel wurde nach der Attestierung der akademischen Leistungen das kantonale Examen für ein Lehrpatent erwartet. Abweichungen von diesem Modell fanden sich vor allem in Basel-Stadt, wo nach dem Universitätsstudium noch ein zweisemestriger Kurs am Lehrerseminar zu absolvieren war, und in St. Gallen, das eine eigene Sekundarlehramtsschule führte und diesbezüglich keine enge Kooperation mit der Hochschule St. Gallen pflegte.

Wie vielgestaltig und kantonal different die Ausbildungsgänge für die Volksschuloberstufe jahrzehntelang blieben, demonstriert ein Bericht des Erziehungsrats des Kantons Luzern aus dem Jahr 1984, der skeptisch gegenüber Harmonisierungsbestrebungen von Lehrpersonenausbildungen ist:

Wie können die effektiv bestehenden Ausbildungsgänge für Reallehrpersonen und Sekundarlehrer praktisch zusammengeführt werden, dass nur noch typenspezifische Akzentuierungen da sind? Geht es da nicht letztlich um indirekte Abschaffung der Reallehrerbildung? Freiburg z.B. kümmert sich vorderhand nicht um die Ausbildung der Reallehrer, in Bern werden keine Reallehrer ausgebildet, in St. Gallen und Zürich sind die Ausbildungsgänge für Sekundarlehrer und Reallehrer sehr unterschiedlich (Erziehungsrat LU, 1984, S. 4). 
Tabelle 1: Übersicht über die Lehrpersonen-Ausbildung für Realschulen, Sekundarschulen und Progymnasien (Stand 1965). Quelle: Archiv für das schweizerische Unterrichtswesen 1965/66

\begin{tabular}{|c|c|c|}
\hline Kantone & Institution der Lehrerbildung; Dauer der Ausbildung & Aufnahmebedingung \\
\hline \multirow[t]{3}{*}{$\mathrm{ZH}$} & $\begin{array}{l}\text { Sekundarlehramt; viersemestriges Studium an der } \\
\text { Universität }\end{array}$ & $\begin{array}{l}\text { Zürcherisches Wählbar- } \\
\text { keitszeugnis als Primarlehrer }\end{array}$ \\
\hline & $\begin{array}{l}\text { Fachlehrerpatent; viersemestriges Studium an der } \\
\text { Universität }\end{array}$ & k.A. \\
\hline & $\begin{array}{l}\text { Lehramt für Real- und Oberschule im kantonalen } \\
\text { Seminar zur Ausbildung für die Real- und Oberschule; } \\
\text { zwei Jahre }\end{array}$ & $\begin{array}{l}\text { Fähigkeitszeugnis als } \\
\text { zürcherischer Primarlehrer, } \\
\text { zweijähriger erfolgreicher } \\
\text { Unterricht }\end{array}$ \\
\hline $\mathrm{BE}$ & $\begin{array}{l}\text { Sekundarlehramt; fünfsemestrige Ausbildung an der } \\
\text { Lehramtsschule Bern in Verbindung mit den philoso- } \\
\text { phischen Fakultäten der Universität }\end{array}$ & $\begin{array}{l}\text { Primarlehrerpatent und } \\
\text { einjähriger Schuldienst; für } \\
\text { Maturanden Besuch eines } \\
\text { Vorkurses }\end{array}$ \\
\hline $\mathrm{LU}$ & $\begin{array}{l}\text { Keine Sekundarlehramtsschule; aber eigene Sekun- } \\
\text { darlehrerprüfungen; viersemestriges Studium an einer } \\
\text { Hochschule }\end{array}$ & $\begin{array}{l}\text { Primarlehrerpatent oder } \\
\text { Maturitätszeugnis }\end{array}$ \\
\hline UR & Ausbildung ausserhalb des Kantons & k.A. \\
\hline SZ & $\begin{array}{l}\text { Ausbildung ausserhalb des Kantons; viersemestriges } \\
\text { Studium an einer Hochschule oder Sekundar- } \\
\text { lehramtsschule }\end{array}$ & $\begin{array}{l}\text { Primarlehrerpatent oder } \\
\text { Maturitätszeugnis mit } \\
\text { Mindestnote } 4,5\end{array}$ \\
\hline $\begin{array}{l}\text { OW; NW; } \\
\text { GL; ZG }\end{array}$ & Ausbildung ausserhalb des Kantons & k.A. \\
\hline FR & $\begin{array}{l}\text { Les maîtres de l'ênseignement secondaire sont formés à } \\
\text { l'Université; quatre semestres }\end{array}$ & $\begin{array}{l}\text { Maturité ou brevet suisse de } \\
\text { maître primaire }\end{array}$ \\
\hline $\mathrm{SO}$ & k.A. & k.A. \\
\hline BS & $\begin{array}{l}\text { Lehramt an mittleren und oberen Schulen und } \\
\text { Fachlehramt; zweisemestrige Kurse zur pädagogischen } \\
\text { Ausbildung am kantonalen Lehrerseminar (am pädago- } \\
\text { gischen Institut) }\end{array}$ & $\begin{array}{l}\text { Maturitätszeugnis oder von } \\
\text { der Universität anerkannter } \\
\text { Fähigkeitsausweis; an der } \\
\text { Universität oder an einer } \\
\text { Fachschule bestandene } \\
\text { Fachprüfung }\end{array}$ \\
\hline $\mathrm{BL}$ & k.A. & k.A. \\
\hline $\mathrm{SH}$ & $\begin{array}{l}\text { Reallehramt (Sekundarlehramt); Studium an einer } \\
\text { Hochschule oder Sekundarlehramtsschule }\end{array}$ & Elementarlehrerpatent \\
\hline AR; AI & Ausbildung ausserhalb des Kantons & k.A. \\
\hline SG & $\begin{array}{l}\text { Sekundarlehramtsschule in St. Gallen; fünfsemestriges } \\
\text { Studium }\end{array}$ & $\begin{array}{l}\text { Maturitätszeugnis oder } \\
\text { erstklassiges Primarlehrer- } \\
\text { patent }\end{array}$ \\
\hline GR & k.A. & k.A. \\
\hline \multirow[t]{2}{*}{ AG } & $\begin{array}{l}\text { Sekundarlehrerausbildung; zweisemestriges Studium im } \\
\text { französischen Sprachgebiet }\end{array}$ & $\begin{array}{l}\text { Primarlehrerpatent mit } \\
\text { qualifizierter Note }\end{array}$ \\
\hline & $\begin{array}{l}\text { Wahlfähigkeit für Bezirksschulen; dreijähriges } \\
\text { Hochschulstudium }\end{array}$ & $\begin{array}{l}\text { Maturitätszeugnis oder } \\
\text { Primarlehrerpatent }\end{array}$ \\
\hline TG & $\begin{array}{l}\text { Sekundarlehrerpatent; Ausweis über ein viersemestriges } \\
\text { Studium }\end{array}$ & $\begin{array}{l}\text { Primarlehrerpatent oder } \\
\text { Maturitätszeugnis }\end{array}$ \\
\hline
\end{tabular}


Derartige Aussagen kommentierte Anton Hügli Ende der 1980er-Jahre wie folgt:

Schweizerischer Realismus, Prestigedenken und Standesdünkel haben dazu beigetragen, dass diese Spaltung in universitäre und nichtuniversitäre Lehrerbildung nie ernsthaft in Zweifel gezogen worden ist. Die Sekundarschule, die zu den höheren Schulen hinführen soll, ist innerhalb des mehrgliedrigen Schulsystems selbstverständlich der vornehmste, begehrteste Typus, demgegenüber der mittlere Typus, der in erster Linie zur Berufslehre hinführt, oder gar der dritte Typus mit den leistungsschwächeren Schülern schon kaum mehr zählt. Für die Lehrer dieses dritten Typus, die in aller Regel auch als Allrounder, als Lehrkräfte für alle Fächer, eingesetzt werden, ist in beinahe allen Kantonen das Primarlehrerdiplom, ergänzt um einige Weiterbildungskurse, als Ausbildung nach wie vor gut genug. Nur in einigen wenigen Kantonen, St. Gallen, Zürich und Luzern zum Beispiel, gibt es neuerdings auch für diese Lehrkräfte eigene Lehrerbildungsinstitute (Hügli, 1989, S. 352).

Auch Mitte der 1990er-Jahre hatte sich in der deutschsprachigen Schweiz noch keine stufenbezogene Ausbildung für Lehrpersonen der Sek I durchgesetzt, so dass die in der Tabelle dargestellte Ausbildungssituation von Mitte der 1960erJahre im Wesentlichen auch 20 bis 30 Jahre später gültig war. Lehrerinnen und Lehrer für den Leistungszug mit Grundanforderungen absolvierten nach dem Primarlehrerpatent eine Zusatzausbildung zum Reallehrer - ausser in Zürich, dort gab es eine eigenständige nachmaturitäre Ausbildung für Reallehrpersonen. Gegen Mitte der 1970er-Jahre erfolgte in vielen deutschsprachigen Kantonen die Streckung der Ausbildungszeit im Seminar von vier auf fünf Jahre. Damit wurde der Weg für einen Hochschulzugang der Absolvierenden (zumeist an eine philosophische Fakultät) geschaffen (Criblez, 2010). Lehrpersonen, die in einer Sekundarschule oder in einer progymnasialen Schule bzw. Bezirksschule unterrichten wollten, mussten ein Hochschulstudium oder in St. Gallen die Sekundarlehramtsschule absolvieren.

\section{Bildungspolitische Konfliktlinien zur Sekundarstufe I}

\section{Gremien und bildungspolitische Fragen zur Lehrperso- nenausbildung Sek I}

Ein wesentlicher Impuls für das Überdenken der tradierten Strukturen der Volksschule und der damit zusammenhängenden Ausbildungswege für Lehrpersonen ging von dem 1975 veröffentlichten Bericht «Lehrerbildung von morgen» (LEMO) aus. Die im Auftrag der EDK agierende Expertenkommission hatte sich zum Ziel gesetzt, «über die Einigung auf den Bildungsauftrag und auf das Bildungsprogramm der schweizerischen Lehrerbildungsanstalten eine Verbes- 
serung und eine Harmonisierung der künftigen Lehrerbildung in der Schweiz (zu) erreichen» (LEMO, 1975, S. 15). Die Ausführungen des Berichts zielen gesamthaft auf die Ausbildung der Lehrpersonen für die Primarstufe. Aussagen zur Lehrpersonenausbildung für die Sekundarstufe befassen sich hauptsächlich mit der Lehrerweiterbildung, so z.B. das Kapitel 4.2 «Die Weiterbildung zum Lehrer für die Sekundarstufe I» (LEMO, 1975, S. 259 ff.). Der Bericht hält fest, dass für die Weiterbildung zum Lehrer der Sek I eine mit dem Primarstufenlehrer gemeinsam absolvierte Grundausbildung von ein bis zwei Jahren vorausgesetzt werde. Anschliessend war eine schulartspezifische Ausbildung vorgesehen sowohl für Lehrpersonen der abschliessenden Schulen, die ihre Schülerinnen und Schüler ins Berufsleben entlassen, als auch für Lehrpersonen der weiterführenden Schulen, welche auf die Mittelschulen und anspruchsvollen Berufslehren vorbereiten. Bereits im LEMO-Bericht schlug 1975 die Expertengruppe eine neu zu schaffende Studienkommission vor, die Koordinationspläne für die Lehrerbildung der Sek I weiterentwickeln und spezifizieren sollte (LEMO-Bericht, 1975).

Ein derartiges Gremium wurde mit der Plenarversammlung der EDK 1978 als Ausschuss Lehrerbildung (ALB) der Pädagogischen Kommission und der Mittelschulkommission eingerichtet. Als eine Art Unterausschuss des ALB verfolgte die Arbeitsgruppe Sekundarstufe I (AGS I) seit Ende 1980 den EDK-Auftrag und legte 1982 den Schlussbericht mit dem Titel «Die Ausbildung der Lehrer für die Sekundarstufe I» vor. Ziel dieses prospektiven Berichts, der bei vielen Vernehmlassungspartnern Konflikte auslöste und auf starke Ablehnung stiess, war es, angesichts der Ungleichartigkeit der Ausbildungsmodelle für Lehrpersonen der Volksschuloberstufe und der verschiedenen kantonalen Reformprojekte den Planungsstellen der Erziehungsdepartemente und Bildungsdirektionen eine bildungspolitische Rahmung zu geben, von der koordinative Wirkung ausgeht.

Bildungspolitische Konflikte können zu zentralen Antriebskräften von Reformen der Lehrpersonenausbildung werden. Sie geben Auskunft über Problemlagen, «innere Widersprüche» oder Einflussunterschiede von Anspruchsgruppen. Die in diesem Beitrag angesprochenen Konflikte sollen als eine Form bildungspolitischer Kommunikation von Akteuren verstanden werden, die neben der Option Konflikt auch Kooperation oder Konkurrenz als Strategien verfolgen konnten (Raschke \& Tils, 2007, S. 225). Folgende bildungspolitische Fragen und Konflikte, die jahrzehntelang kaum reguliert werden konnten, gelangten Ende der 1970er-Jahre u.a. in die bildungspolitische Arena:

- Darf durch die Reorganisation von Ausbildungsmodellen für Lehrpersonen der Versuch unternommen werden, Erneuerungen der Organisation der Volksschuloberstufe einzuleiten, oder ist jedes schulplanerisch präjudizierende Moment zu vermeiden und Lehrpersonenausbildung an vorherrschenden Schulstrukturen auszurichten (Egger, 1983, S. 25)? 
- Sollten für die Volksschuloberstufe Lehrpersonen mehrheitlich mit dem Selbstverständnis als «Allrounder» oder als «Fachgruppenlehrer» ausgebildet und beschäftigt werden (Egger, 1983, S. 164; Rickenbacher, 1975, S. 42)?

- Ist die Frage nach konsequenter «Akademisierung», «Verwissenschaftlichung» und «Tertiarisierung» der Ausbildung für Lehrpersonen der Volksschuloberstufe eine Frage der Professionalisierung, eine Statusfrage oder eine ideologische Frage (Gehrig, 1999, S. 96)?

- Sollte die Lehrpersonenausbildung typen- oder stufendifferenziert aufgebaut sein (Gehrig, 1999, S. 104)?

Weitere Fragen bezogen sich auf die Integration der Fächer Handarbeiten/ Werken und Hauswirtschaft in allfällig tertiarisierte Ausbildungsmodelle sowie auf ein Doppelpatent für die Sek I und Sek II. Konfliktlinien, wie sie sich in solchen Frageensembles spiegeln können, wirkten als Hintergrundfaktor auf die Diskussionen und Formulierungen von Gremien wie z.B. die AGS I und ihr Prospektivbericht, ohne dass es zwangsläufig zu anderweitigen «Konfliktmanifestationen» kommen musste. Die AGS I selbst war ein wissenschaftlich orientiertes Kräftefeld im Sinne eines Mikrokosmos, welches nach der Beauftragung durch die EDK sich relativ autonom entwickeln konnte (Bourdieu, 1998, S. 19). Von diesem Set an Konfliktlinien werden in den folgenden Abschnitten der Konflikt um die Akademisierung und der um die Typen- oder Stufendifferenzierung der Lehrpersonenbildung für die Sek I quellen- und dokumentennah näher beleuchtet:

\section{Akademisierung und Ort der Lehrpersonenausbildung als}

Thema einer Konfliktlinie

Während der LEMO-Bericht sowohl den seminaristischen als auch den maturitätsgebundenen Weg in der Grundausbildung für Lehrpersonen der Primarstufe im Sinne der gesamten Volksschulzeit als zwei gleichgewichtige und wünschbare Formen bezeichnete (LEMO-Bericht, 1975) - und mit der Situation der Unentschiedenheit die zu erwartende Beratungsfunktion von einem Expertengutachten gegenüber bildungspolitischen Akteuren nur unzureichend erfüllte (Criblez, 2008; Messmer, 1999) -, war die Empfehlung des Prospektivberichts eindeutiger. Die postmaturitäre Struktur des vorgeschlagenen Ausbildungsmodells vorausgesetzt, plädierte die AGS I dafür, die Ausbildung für alle Lehrpersonen der Sek I an einem gemeinsamen, im Rahmen einer Universität selbstständigen Institut erfolgen zu lassen (Egger, 1983). Aus der Retrospektive wird deutlich, dass mit diesem Vorschlag eine "Zumutung» gegenüber dem Status Quo von Ausbildungsmodellen für die Primarstufe im Sinne der Volksschule, die im System der Sek II angesiedelt waren, ausgesprochen wurde: Für seminaristische Ausbildungsformen bedeutete der Tertiarisierungsvorschlag in Richtung Universität einen beachtlichen Sprung in der zeitlichen Ausdehnung der Ausbil- 
dungsstruktur (Gehrig, 1999). Dennoch ist es plausibel, dass die AGS I nicht eine andere "tertiäre Verortung» vorgeschlagen hatte. Mit diesem Vorschlag wurde lediglich das Interesse von Reallehrpersonen tangiert, die vom bisherigen Seminar-Modell weiterhin überzeugt waren. Eine andere tertiäre Verortung hätte neben dem Interesse einer Gruppe von Reallehrpersonen auch noch die Interessenssphäre von Lehrpersonen der Sekundarschulen bzw. Schulen mit Mittelschulanschluss berührt. Da diese Lehrpersonengruppe - wie Tabelle 1 es zeigt - in der Regel ein sechssemestriges Studium an einer Universität absolvierte, hätte die Zuordnung zu einer nichtuniversitären Ausbildungsinstitution eine Herauslösung der progymnasialen Lehrpersonen aus dem bereits erreichten Status bedeutet (Gehrig, 1999) - wie sie in der deutschsprachigen Schweiz mit Gründung der pädagogischen Hochschulen (mit Ausnahme des Kantons Freiburg) dann auch tatsächlich vorgenommen wurde.

Welche Haupt-Argumentarien lassen sich bei einer inhaltsanalytischen Sichtung der Vernehmlassungsrückmeldungen - als Auswahl dargestellt in Tabelle 2 - herauspräparieren? Und: Wie können diese im Spiegel heutiger Forschungsergebnisse bewertet werden?

\section{Tabelle 2: Vernehmlassungsbemerkungen zur Neufassung der Lehrerausbildung und zu den Schulstrukturen; Quelle: StA LU, A 1270/836; A 1270/837, (1984)}

\begin{tabular}{|l|l|}
\hline \multicolumn{2}{|c|}{ Vernehmlassungsbemerkungen zum } \\
Ausbildungsinstitut
\end{tabular}


"Eine verbesserte Qualifikation wird (...) nicht in erster Linie durch einen akademischen Ausbildungsgang erreicht, sondern vielmehr sollte den Lehrerstudenten vermehrt Gelegenheit gegeben werden, zu verstehen und entsprechende Handlungsmöglichkeiten zu überprüfen bzw. einzuüben. (...) Die Universitäten haben bis heute den Beweis nicht erbracht, dass sie in der Lage sind, eine volks- und praxisnahe Lehrerausbildung zu garantieren. Von den im Bericht aufgeführten Absichtserklärungen halten wir nicht sehr viel. Lehrerbildung ist nur denkbar in relativ kleinen Gruppen, nicht aber im Rahmen der Masseninstitution Universität, wo die Lehrerbildung ein bloss geduldetes Anhängsel war, ist und sein wird. (...) Eine generelle Intellektualisierung der Lehrer ist daher abzulehnen» (2).

"Nirgends, ausser im Projekt der Zentralschweiz und zum Teil in St. Gallen, wird die Reallehrerbildung ernsthaft in eine sehrerbildung der Sekundarstufe I> einbezogen. (...) $\mathrm{Zu}$ relativieren ist die Forderung nach Eingliederung der Ausbildung in eine Universität. Der Bericht legt den Primat auf die Berufsausbildung (gegenüber der akademischen Grundausbildung). Er schlägt klar gegliederte, (verschulte` Studiengänge vor, betont den Praxisbezug und den Bezug zur ausserschulischen Berufs- und Arbeitswelt und sieht keine eigentlichen Forschungselemente im Studium vor. Das alles ist gerade nicht typisch für die Universität. Gegen eine Integration eines selbständigen Instituts in eine Universität ist dann nichts einzuwenden, wenn der Theorie-Praxis-Bezug wirklich ernst genommen wird. (...) Die Grösse des Instituts müsste beschränkt werden. Die Stellung des Institutes müsste im Rahmen der Gesamtuniversität verbessert werden" (4).

«Die Volksnähe (Volksverbundenheit) des zukünftigen Lehrers muss gewährleistet sein. (...) Wirtschaft, Leben und Schulalltag sollen in der Ausbildung verstärkt beachtet werden. Das Ausbildungsinstitut soll eine überschaubare Grösse besitzen und auf die Regionen verteilt angesiedelt werden. (...) Die Ausbildung der Lehrer für die Sekundarstufe I erfolgt an einem gemeinsamen, selbständigen Institut mit Hochschulniveau» (3).

«Die Angliederung der Lehrerausbildung an eine Universität scheint uns nicht gegeben zu sein; weil z.B. die PHS in St. Gallen allen Anforderungen einer guten Lehrerbildungsstätte nachkommt» (1).

«Eine hohe Ausbildungsqualität wird nicht nur durch ein Universitätsstudium erreicht. Regionale Ausbildungsstätten mit universitärem Charakter können diese Aufgabe ebenso gut erfüllen. Ein Universitätsstudium bringt für Nichthochschulkantone grosse Probleme. Die Verbindung des Studenten zur Region geht verloren, die gewünschte «Volksnähe» ist nicht zu verwirklichen. Der Unterricht an der Ausbildungsstätte soll beispielhaft für das spätere Unterrichten an der Volksschule sein. Sind Universitäten dazu in der Lage ?» (2)

In mehreren Stellungsnahmen wird das Argument aufgebaut, dass eine allfällige Studienorganisation an Universitäten einen tendenziell verakademisierten, praxisuntauglichen und damit vom "Volk» abgehobenen Habitus heranbilde (Hoffmann-Ocon \& Metz, 2010). Als Kondensat dieser Auffassung kann der Satz «Eine generelle Intellektualisierung der Lehrer ist daher abzulehnen» (ED Zug, 1984, S. 2) gelten. Auch wenn der Begriff «Intellektualisierung» einen hermeneutisch schwer auflösbaren Rest in sich birgt, zielt er konnotativ auf eine wissenschaftsskeptische Orientierung. Diese basiert auf der Ablehnung eines 
Habitus forschenden Lernens für Lehrpersonen. Eine derartige Orientierung stilisiert den vortheoretischen Zugang zum pädagogischen Handeln und suggeriert, dass der Zirkel des rein Praktischen nicht durchbrochen werden könne, weshalb auf praktische Probleme praktisch reagiert werden müsse. Das implizierte handwerklich-praktische Modell des Theorie-Praxis-Bezugs konstruiert die Lehrperson «als den erfahrenen Praktiker und Routinier» (Duncker, 2002, S. 23). Obgleich die Lehrerseminare in der deutschsprachigen Schweiz seit Beginn des 20. Jahrhunderts nicht nur Orte der Wissensdistribution, sondern auch Orte der Wissens- und Theorieproduktion waren (Grunder, 1993), wird mit einigen Ausnahmen (z.B. Freiburg und Luzern) die Verlegung des Ausbildungsortes an die Universität abgelehnt. Die Positionierungen betreffend Tertiarisierung sind dagegen ambivalenter. Mehrere Vernehmlassungspartner sprechen sich nicht gegen eine Ausbildung auf Hochschulniveau aus. Als idealer Typus wird eine «volksnahe» Lehrperson entworfen, die an den paidotropen, an «Unterrichtsfragen vom Blickwinkel des Kindes» und jugendpsychologisch interessierten Schulmann erinnert und im Gegensatz zu dem logotropen Fachwissenschaftler im Unterrichtszimmer steht, ganz so, wie Christian Caselmann (1964) im Zuge einer - aus heutiger Sicht zweifelhaften - Ausdruckspsychologie mit seiner Typenlehre die "Wesensformen des Lehrers» beschrieb. Die Tertiarisierungsbemühungen konnten tatsächlich also mit Bezug auf wissenschaftliche Kapitalsorten, die sich in einem historischen Prozess vom reinen wissenschaftlichen in ein institutionalisiertes Kapital gewandelt haben, «bezweifelt» werden (Bourdieu, 1998, S. 32).

Bildungssoziologische und erziehungswissenschaftliche Studien der letzten Jahre gelangen zu dem Befund, dass der 1983 teilweise geforderte Idealtyp des Lehrerinnen- und Lehrernachwuchses angesichts heutiger Professionalisierungsanforderungen höchst defizitär erscheint. Während seinerzeit Volksnähe und Skepsis gegenüber Intellektualisierung als wünschenswerte Charakterisierung der Lehrperson im diskursiven Feld der Lehrerinnen- und Lehrerbildung vorherrschte, wird aktuell die Theorieferne und Bildungsmüdigkeit von angehenden Lehrpersonen im Rahmen der «Selbstselektion» moniert. Demnach interpretieren Studierende die im Vergleich mit universitären Hochschulen geringere Reputation und geringere wissenschaftliche Qualifikation des Personals der pädagogischen Hochschulen dahingehend, dass auch der wissenschaftliche Anspruch tiefer sei (Denzler \& Wolter, 2008). Stefan Denzler und Stefan C. Wolter gelangen in ihrer Studie zur «Selbstselektion bei der Wahl eines Lehramtsstudiums» zu dem Befund, dass die Studierenden für Studiengänge der Volksschule an pädagogischen Hochschulen in der deutschsprachigen Schweiz zum einen eher eine Sozial- und Familienorientierung anstreben und weniger an Wissenschaft und Wissenserwerb interessiert seien und zum anderen sich umso stärker für ein Studium an einer pädagogischen Hochschulen interessieren, je entfernter sie zu einer Universität wohnen (Denzler \& Wolter, 2008). Aus bildungshistorischer Sicht überraschen derartige Erkenntnisse wenig. Bereits 
Ende der 1960er-Jahre kommt die empirische sozialpsychologische Untersuchung von Gerd Mietzel zu ähnlich lautenden Ergebnissen. Oberprimaner (Abiturienten in Deutschland) schätzten gemäss eines Stereotyps zu Volksschullehrpersonen diese Professionsvertreter dergestalt ein, dass diese im Unterricht eher das Kind als den Unterrichtsstoff beachteten, dass sie keine Theoretiker seien und ihnen mehr das praktische Denken sowie Handeln liege und dass für die wissenschaftliche Arbeit ihnen der Ehrgeiz fehlen dürfte (Mietzel, 1967). Insgesamt liegt die Vermutung nahe, dass in etlichen Vernehmlassungsrückmeldungen auch von Vertretern der Bildungsadministration stereotype, tradierte Vorstellungen über den Ort der Lehrpersonenausbildung eingeflossen sind, die einem Bildungsgang für Lehrpersonen an Universitäten absprechen, den - ohnehin schwer bestimmbaren - Praxisbezug herstellen zu können. Demgegenüber dominiert heute der Verweis auf Professionsmerkmale des Lehrberufs, die z.B. in der Autonomie und der Wissensbasis der Berufsausübung liegen. Letztere grenze den Lehrberuf von handwerklichen und gewerblichen Berufen ab. Das professionelle Wissen ziele auf abstraktes Wissen, welches in der Regel durch eine akademische Ausbildung eingelöst werde, nicht nur in den Unterrichtsfächern, sondern auch in den für die Vermittlungstätigkeit relevanten Disziplinen (Gensicke, 2006; Herzog, 2011). Auffallend ist auch, dass in den Vernehmlassungsrückmeldungen neben ausbildungsinhaltlichen und konzeptionellen Begründungen ebenso bildungspolitisch-formale, wie etwa das Argument der Schlechterstellung von Nichthochschulkantonen, genannt wurden.

\section{Orientierung der Ausbildung an Schultypen oder Schulstufen?}

Bereits Ende der 1960er und Anfang der 1970er-Jahre wurden Zweifel an der traditionellen Schultypengliederung der Sek I aus der wissenschaftlichen Gemeinschaft laut. Auch in der deutschsprachigen Schweiz wurden Schulreformen diskutiert, die auf eine Gesamtschulorganisation zielten (Haeberlin, 1972a; Haeberlin, 1972b). Als eine Art Experimentalprogramm konnte lediglich die Gesamtschule Dulliken eingerichtet werden, die von 1970 bis 1980 Bestand hatte (Jenzer, 1983). Dabei bleibt bemerkenswert, dass die Frage nach einer Gesamtschul-Lehrpersonenbildung explizit nicht gestellt wurde. Spätere kantonale Reformen mit Gesamtschul-Elementen wie etwa das Zürcher AVO-Modell (Abteilungsübergreifende Versuche an der Oberstufe) wurden auf ihre Kompatibilität mit den vorherrschenden Ausbildungsmodellen untersucht (Criblez \& Bosche, 2007; Gerber, 1987). Der Vernehmlassungsbericht der AGS I schlug eine stufenspezifische Grundausbildung der Studierenden vor, die typenspezifische Differenzierungen aufweisen durfte. Aber: «Die gemeinsamen Gegebenheiten der Sekundarstufe I (...) sind (...) wesentlich bestimmender für die Lehrerbildung als spezifische Ausprägungen der Schultypen. Wir messen deshalb der typenspezifischen Differenzierung nur als Mittel der Akzentuierung, nicht aber der Separierung der Studiengänge einen Eigenwert bei» (Egger, 1983, 
S. 125). In der Tabelle 3 werden drei ausgewählte Vernehmlassungsrückmeldungen von kantonalen Lehrerkonferenzen bzw. Lehrerverbänden aufgeführt, um die Orientierungen der Berufsfeldakteure im Konsultationsprozess hervorzuheben.

\section{Tabelle 3: Vernehmlassungsbemerkungen zur Neufassung der Lehrerausbildung und den Schulstrukturen; Quelle: StA LU, A 1270/836; A 1270/837, (1984).}

Vernehmlassungsbemerkungen zur typenspezifischen Ausbildung

"Aus der Beobachtung heraus, dass z.B. ein Stadt-Zürcher-Realschüler nicht mit einem solchen aus dem St. Galler Rheintal verglichen werden kann, scheint uns die Fähigkeit des Lehrers, sich mit regionalen Besonderheiten des entsprechenden Schülertyps vertraut zu machen, besonders wichtig zu sein. (...) Obwohl in einem früheren Kapitel dargelegt wurde, dass es problematisch sei, Schüler nach lern- und schulerfolgsrelevanten Merkmalen kategorisieren zu wollen, fragen wir uns doch, ob ein Lehrer das ganze Spektrum vom schwachen, langsam lernenden Realschüler bis zum Spitzensekundarschüler abdecken kann. Wir glauben, dass dies nicht möglich ist» (2f.).

«Der Bericht zeichnet das Bild des Schülers, der heute sowohl in der Realwie auch in der Sekundarschule bestehen kann. Daneben scheint uns aber, dass der typische Realschüler und der typische Sekundarschüler ebenso beschrieben werden können. (...) Die heutigen Gesellschaftsstrukturen sind nicht geeignet, die bestehenden Unterschiede zwischen diesen Schülertypen zu eliminieren. Die Berichterstatter verlangen vom Lehrer nach unserer Meinung unrealistisch hohe Fach- und Handlungskompetenzen. Der Lehrer als menschlicher Bezugspartner des Schülers wird im Bericht zu wenig erwähnt» (1 f.).

«Es ist eine Tatsache, dass der Hauptharst der Schüler der Sek. und OS (Kt. Solothurn) aus einem anderen sozialen Milieu herauswächst. Daraus ergibt sich ein zum Teil anderes soziales Verhalten, Sprachverhalten» (1).
Kantonale Sekundarlehrerkonferenz St. Gallen

Reallehrerkonferenz

Kanton Thurgau
Sekundarlehrer-Verein des Kantons Solothurn

In der Tendenz wurde mit den Vernehmlassungsrückmeldungen der Versuch unternommen, die verschiedenen tradierten Ausbildungsmodelle für Lehrpersonen der Volksschuloberstufe damit zu begründen, dass es Schülertypen mit unterscheidbaren Merkmalen gebe, für die spezielle Schultypen vorgehalten werden müssten. Insbesondere die Frage nach der typenspezifischen Ausbildung von Lehrpersonen berührte eine bis heute aktuelle Debatte, welche spezielle Schülerinnen- und Schüler- sowie Lehrpersonentypologien implizierte. In der deutschsprachigen Schweiz war Konrad Widmer ein Protagonist der Deutungsproduktion von Argumentarien zur Beibehaltung von Schultypen auf der Volksschuloberstufe. Zum Schlüsseltext wurde der Beitrag «Braucht der Realschüler eine eigene Schule?», welcher 1980 in der Rundschau der Abschlussklassenlehrer erschien, dem Mitteilungsblatt der Arbeitsgemeinschaft der Abschlussklassenlehrer des Kantons St. Gallen. Der Artikel selbst beruhte auf einem Vortrag. Diese Details sind rekonstruierbar, weil die Mitglieder des EDK-Gremiums 
AGS I sich durch die publizierten Überlegungen bildungspolitisch herausgefordert sahen (StA LU, A 1270/834, Hedinger, 1981). In seinem Artikel stellte Widmer u.a. die Frage, ob der Realschüler ein besonderer Typ von Schüler sei, der einen eigenen Schultypus rechtfertige. Aus der Perspektive der Pädagogischen Psychologie grenzte Widmer seine Position von der Begabungsvorstellung der Frankfurter Schule ab, nach der Begabung ein Produkt unterschiedlicher Lern- und Entwicklungsanreize in verschiedenen Schichten bzw. Klassenlagen sei. Mit Hilfe der Humangenetik, so führte Widmer aus, hätte die Psychologie die Einsicht gewonnen, dass für Befähigungen genetische Strukturen vorlägen (StA LU, A 1270/834, Widmer, 1980). Zwar schränkte Widmer seine eher anthropologische Aussage etwas ein, indem er behauptete, dass die effektive Leistungsfähigkeit in einem Lern- oder Verhaltensbereich immer das Produkt und nicht die Summe der Erbinformationen sowie der Anregungspotenz der Umwelt sei, aber er konstatierte unterschiedliche Intelligenztypen bei Schülern, die unterschiedliche Schultypen legitimierten:

Die Tatsache, dass in der Realschule eher Schüler aus mittleren und unteren Sozialschichten stammen, lässt erklären, dass die Leistungs- und Verhaltensstruktur dieser Schüler in die Richtung der anschaulich-praktischen Intelligenz und wohl etwas weniger in die Richtung der begrifflich-theoretisch-sprachlichen Intelligenz tendiert. Möglich sind auch einseitige Begabungen. (...) Die anschaulich-praktische Tendenz im Lern- und Verhaltensspielraum darf nicht geringer geachtet werden als die theoretisch-begriffliche. (...) Wir sind der Ueberzeugung, dass aus den angeführten Gründen ein besonderer Schultypus Realschule gerechtfertigt ist (StA LU, A 1270/834, Widmer, 1980, S. 35 f.).

Für Widmer folgte aus der Begründung des Schultypus Realschule, dass diese angesichts der Diskussion um didaktische Modelle (Anfang der 1980er-Jahre) ein eigenes noch zu entwerfendes didaktisches Konzept bräuchte. Die in der Tabelle 3 angeführten Vernehmlassungsrückmeldungen zeigen in der Tendenz deutlich, dass die Repräsentanten des Praxisfeldes Widmers Typologie des Realschülers folgten und eine weiterhin schultypbezogene Lehrpersonenausbildung als plausibel erachteten. Widmer bot den Akteuren der Standesvertretungen «reines wissenschaftliches Kapital» zur Abwehr von Reformen der Ausbildungsstrukturen an.

Demgegenüber argumentierten die Verfasser und Befürworter des Prospektivberichts, dass die grundsätzlichen Unterschiede zwischen den Schülertypen - die je spezifische Lehrpersonenausbildungsmodelle begründeten - empirisch schwer nachweisbar sind. An dem für die Lehrerbildung folgenreichen Typologieansatz wurde kritisiert, dass die Schülermerkmale als weitgehend stabil betrachtet würden, so dass mittels einer genauen Diagnostik und Prognostik die Selektion in die Schultypen der Volksschuloberstufe gelänge. Die Annahme der Merkmalskonstanz wurde mit dem Hinweis bezweifelt, dass die Merkmalsverteilungen bzw. effektiven Leistungen von Schülerinnen und Schülern, welche 
in unterschiedliche Schultypen selegiert wurden, sehr stark überlappen. Dieser Hinweis beruhte auf Befunde der 1977 durchgeführten Studie von Peter Halbherr (1980) im Kanton Baselland. Der Untersuchung zufolge überschneiden sich die mit Tests gemessenen Schulleistungen stark - es wurden ein Rechtschreibtest, ein Rechentest sowie ein Intelligenztest (Prüfsystem für Schul- und Bildungsberatung (PSB)) mit Schülerinnen und Schülern der Realschule, der Sekundarschule und des Progymnasiums durchgeführt. Konkret bedeutete dies, dass die Schülerinnen und Schüler der Realschule zwar nicht den gleich hohen Durchschnittswert wie diejenigen der Sekundarschule hatten, aber die meisten sich im Bereich des Leistungsspektrums der Sekundarschülerinnen und -schüler wieder fanden. Die leistungsschwächsten Sekundarschülerinnen und -schüler lagen sogar markant unter dem mittleren Wert der Realschülerinnen und -schüler (StA LU, A 1270/834, Hedinger, 1981). Zu ähnlichen Ergebnissen gelangte eine Zürcher Studie (Haefeli, Schräder \& Häfeli, 1979). Die im Bildungsbericht Schweiz 2010 angeführten Untersuchungen zur Strukturvielfalt auf der Sek I weisen in dieselbe Richtung wie die über 30 Jahre alte Studie von Halbherr. Die kantonalen Zusatzstichproben von PISA 2006 deuten darauf hin, «dass die Überschneidungen der Leistungen in den drei PISA-Fachbereichen zwischen den Niveaus relativ gross sind: Die Leistungen einiger Schülerinnen und Schüler aus dem tiefsten Niveau A im Kanton Basel-Landschaft erreichen jeweils über 600 Punkte. Sie schneiden damit besser ab als gut die Hälfte der Schülerinnen und Schüler des leistungsstärksten Niveau P (Moser \& Angelone, 2008)» (Schweizerische Koordinationsstelle für Bildungsforschung (SKBF), Bildungsbericht Schweiz, 2010, S. 94).

Die Verfasser und Befürworter des Prospektivberichts sahen in diesen bis heute empirisch nachgewiesenen Überschneidungen, dass die Grundlage für eine typenspezifische Lehrpersonenausbildung für die Volksschuloberstufe nicht gegeben sei: Wenn die Selektionsentscheide die Tendenz haben, relativ unabhängig von den tatsächlichen Voraussetzungen der Schülerinnen und Schüler sich aus der Retrospektive zu bestätigen, wären «allfällige Schülertypen» mehr als Produkt denn als Voraussetzung der Schulstrukturen, auf die eigenständige Lehrpersonenausbildungen zielen sollen, zu betrachten (StA LU, A 1270/834, Hedinger, 1981, S. 6). Vor diesem Diskussionshintergrund empfahl die AGS I, die Ausbildung für alle Lehrpersonen der Sekundarstufe I an einem gemeinsamen, im Rahmen einer Universität selbstständigen Institut erfolgen zu lassen sowie bestehende getrennte Ausbildungseinrichtungen für Real- und Sekundarlehrpersonen zusammenzulegen und in eine Universität zu integrieren (Egger, 1983). Daran schloss sich die Forderung an, die Lehrberechtigung und vor allem die Praxis der Primarlehrpersonen zwingend auf die Primarstufe zu beschränken. Während der erste Teil der Forderung mit den Studiengängen der pädagogischen Hochschulen seit etwa 2003 als realisiert betrachtet werden kann, unterrichten in einzelnen deutschsprachigen Kantonen nicht zuletzt aufgrund des Lehrpersonenmangels immer noch ausgebildete Primarlehrpersonen auf der 
Sek I und tradieren damit auf der faktischen Ebene das überwunden geglaubte Bild vom Lehrpatent für Klassen 1-9 mit allgemeinen Anforderungen auf der Volksschuloberstufe.

\section{Einfluss tradierter Ausbildungsüberzeugungen auf aktuelle Studiengänge für Lehrpersonen der Sek I} Wie sehr die tradierte Typenstruktur auf die heutigen stufenorientierten Studiengänge der pädagogischen Hochschulen noch Einfluss nehmen kann, zeigt folgender kurzer Problemaufriss: Nachdem an den pädagogischen Hochschulen in der deutschsprachigen Schweiz, die eine Sek-I-Ausbildung anbieten, der Wechsel vom curricularen Modell der Ausbildungssequenzen der Vorgängerinstitutionen zum modularen Modell mit Ausbildungseinheiten mehr oder weniger vollzogen wurde, haben sich sukzessive verschiedene Studiengänge für den Erwerb eines EDK-anerkannten Lehrdiploms etablieren können:

- Der integrierte Studiengang, bei dem fachwissenschaftliche, fachdidaktische, erziehungswissenschaftliche sowie schulpraktische Ausbildungselemente zeitlich parallel und bestenfalls konzeptionell (mit der Zwischenstufe eines Bachelors) einschliesslich des Masterstudiums verflochten sind (EDK Richtlinie 4.2.2.4., 2009).

- Der konsekutive Studiengang, welcher im ersten Studienzyklus ein fachwissenschaftliches Studium in zwei Studienfächern an der Universität oder Musik- bzw. Kunsthochschule vorsieht und im zweiten Studienzyklus die berufsfeldbezogenen Module in den Erziehungswissenschaften, den Fachdidaktiken und den berufspraktischen Studien an einer pädagogischen Hochschule im Programm hat (EDK Richtlinie 4.2.2.4., Art. 5, 3, 2009).

- Der in jüngster Zeit eingerichtete Masterstudiengang für Lehrpersonen mit einem neu- oder altrechtlichen Lehrdiplom für die Vorschul- und Primarstufe bzw. Primarstufe («Studiengang für Zweitstudierende»), deren Leistungen aus der Erstausbildung weitgehend angerechnet werden, so dass diese vor allem fachwissenschaftliche, fachdidaktische und im geringeren Masse erziehungswissenschaftliche und berufspraktische Studien zu absolvieren haben (EDK Richtlinie 4.2.2.4.1, 2010).

Gemäss der Interkantonalen Vereinbarung über die Anerkennung von Ausbildungsabschlüssen aus dem Jahr 1993 (EDK 4.1.1, 2005) gelten die für die Sek I erworbenen Lehrdiplome nicht nur für einen bestimmten Schultyp oder Leistungszweig, sondern beziehen sich auf die gesamte Schulstufe. Die Konzeptionen der Studiengänge an den pädagogischen Hochschulen folgen etwa seit 2002 / 2003 mit ungefähr 20-jähriger «Verspätung» den Vorschlägen der AGS I. Auch wenn sich ein Kausalzusammenhang zwischen dem Schlussbericht von 1983 und den Studiengangsentwürfen der pädagogischen Hochschulen schwer rekonstruieren lässt, darf angenommen werden, dass jenes Argumentarium der AGS I das Denken und Handeln der verantwortlichen Akteure und 
Anspruchsgruppen der Studiengänge für die Sek I geleitet haben. Entscheidungen, ob ein Ausbildungsmodell sich auf einen Schultyp oder auf eine Schulstufe beziehen soll, setzen strategische Reflexionen und Kalkulationen voraus. Hinsichtlich der dynamischen Prozesserwartungen von Akteuren der pädagogischen Hochschulen in Sachen Schulstufen-Entwicklung ist die reale Situation eher statisch. Mit einigen Ausnahmen besitzen die meisten deutschsprachigen Kantone drei- bis vierteilige Sekundarstufen (SKBF, Bildungsbericht Schweiz, 2010). Zwar ist es nicht immer sinnvoll, nur solche Erwartungen zu wecken, die sicher erreichbar erscheinen, da ansonsten gesellschaftliche Mobilisierungs- und Unterstützungsanreize verloren gehen können (Raschke \& Tils, 2007). Jedoch wenn die Lehrpersonenausbildung schulstufenbezogen ist, die Schulstrukturen aber weiterhin durch Schultypengliederung gekennzeichnet sind, besteht die Gefahr einer hermeneutischen Driftzone von Anspruchsgruppen der Lehrpersonenausbildung wie etwa von Akteuren der Bildungsverwaltungen oder auch von Dozierenden der pädagogischen Hochschulen selbst, so dass tradierte Schulstruktur-Überzeugungen eine hohe Geltungsmacht (wieder) erlangen:

Zweitstudierende («Stufenumstieg») - also Studierende z.B. mit Primarlehrerdiplom, die ein Masterstudium für die Sek I absolvieren - könnten als prädestinierte Reallehrpersonen gelten. Regulär bzw. integriert Studierende, die ein Bachelor- und Masterstudium im grundständigen Sinne aufnehmen, könnten als optimal in Sekundarschulen einsetzbar betrachtet werden. Für den progymnasialen Leistungszweig könnten lediglich die konsekutiv Studierenden als geeignet in Betracht gezogen werden. Dass dies keine Überinterpretationen sind, zeigen Pressedebatten auf. So kritisierten Anfang März 2011 Mitglieder der sogenannten «Arbeitsgruppe für eine qualitativ bessere Ausbildung der Sekundarlehrkräfte im Bildungsraum Nordwestschweiz» den integrierten Studiengang dahingehend, dass dieser für drei Unterrichtsfächer in minimal neun Semestern $\mathrm{zu}$ absolvieren wäre und $\mathrm{zu}$ wenig fachwissenschaftliches Wissen vermitteln würde. Einzig der konsekutive Studiengang, der drei Jahre Universitätsstudium in zwei Fächern und eine zweijährige pädagogische Ausbildung an der pädagogischen Hochschule vorsehe, sei den Fachansprüchen angemessen - ganz so, wie er bereits seit 1993 von einer Arbeitsgruppe der EDK Nordwestschweiz (NW-EDK) skizziert wurde (Künzli, 1993; Oelkers, 1992; Wirz, 1993). Daher fordere die Arbeitsgruppe generell, dass die Universität wieder den gesamten Unterbau der Lehrpersonenausbildung begründe (Künzle, 2011). Kritiker der Positionierung dieser Arbeitsgruppe - die sich wissentlich oder unwissentlich an dem rein konsekutiven Ausbildungsmodell der pädagogischen Hochschulen in der Romandie (HEP-BEJUNE und HEP Vaud) orientiert - führen an, dass in der deutschsprachigen Schweiz die Tradition der Sekundarlehrerausbildung mehr als ein Studium von lediglich zwei Unterrichtsfächern vorsehe und die EDK mit 270 bis 300 Kreditpunkte einen Rahmen für das Studium Sek I vorgebe und die Vorschläge der Arbeitsgruppe sich auf ein Gefäss von 330 bis 350 Kreditpunkten belaufen würden (Laur, 2011). 
Erodierende Legitimation von bildungspolitischen

Reformentscheidungen

Mit der Rekonstruktion und ersten analytischen Einordnung des Einflusses tradierter Ausbildungsüberzeugungen wird eine Reformentwicklung angesprochen, die einem in der historischen Bildungsforschung bekannten Muster entspricht: Als Reaktion auf Schulreform- oder Lehrpersonenbildungs-Initiativen und auf erste Realisierungsschritte organisieren sich Reformskeptiker von Lehrerverbänden, Elternorganisationen etc., die eine "Abwehr des ungerechtfertigten pädagogischen Radikalismus» zum Ziel haben (Herrlitz et al., 2005, S. 65). Selbst wenn in der Theorie ein Modernisierungszwang von Reformskeptikern anerkannt wird, geht es in der Praxis weniger um die sachliche Angemessenheit einzelner Reformschritte, sondern vielmehr um die soziale Exklusivität von Schülerinnen- und Schülergruppen oder Lehrpersonen für bestimmte Schultypen. Unterdessen verhält sich die Bildungsadministration in der betreffenden Reformfrage lange reserviert und abwartend, streckenweise sogar widersprüchlich. Dieser Prozess lässt sich nicht nur an Bildungsreformverläufen seit dem Ende des 19. Jahrhunderts zeigen (Herrlitz et al., 2005), sondern auch in aktuellen Bildungsreformversuchen, die von Mobilisierungsstrategien der Öffentlichkeit in Bildungsfragen begleitet werden (Caruso, 2011). Seitdem wissenschaftliche Bildungsexpertise nicht mehr vorrangig in Bildungsdepartementen oder damit beauftragten kantonalen Pädagogischen Arbeitsstellen erzeugt wird, erodiert die Legitimität von bildungspolitischen Reformentscheidungen, wenn sie sich nicht auf extern erstellte Gutachten oder einen starken öffentlichen Konsens abstützen kann. Das Angewiesensein auf externe Legitimationskonstruktionen begünstigt die Reformulierung bekannter Positionen - in diesem Fall Standpunkte zu schultypenorientierten Studiengängen für Lehrpersonen der Sek I.

\section{Resümee: Reformbemühungen der Sek-I-Lehrperso- nen-Ausbildung vor und hinter den Kulissen}

Die Rekonstruktion und Interpretation zur Meinungsproduktion über eine angemessene Reform der Lehrpersonenausbildung für die Sek I zeigt, dass in der deutschsprachigen Schweiz bis zur Einrichtung von diesbezüglichen Studiengängen an pädagogischen Hochschulen von den 1950er-Jahren an «vor den Kulissen» kein grundlegender Systemwechsel stattfand. Als die Meinungsproduktion «leitende Regel» für Lehrpersonen-Ausbildungsmodelle galt die Orientierung an unterscheidbaren Schultypen auf der Volksschuloberstufe. Aus einer (späteren) Sek-I-Optik verlief traditionell die Scheidegrenze zwischen einem umfassend verstandenen Primarlehramt und dem Lehramt für Sekundarschulen mit Mittelschulanschluss, das in den hier vorgestellten Fällen bereits einen akademischen Charakter aufwies. So gehörte z.B. das Berufsverständnis 
des "Allrounders» zur Lehrperson an der Volksschuloberstufe, während Lehrpersonen des progymnasialen Schultyps als Fach- bzw. Fachgruppenlehrpersonen agierten.

Dennoch waren in verschiedenen kantonalen Rahmungen kleinere Reformschritte zu verzeichnen wie etwa der Aufbau einer eigenständigen Ausbildung für Reallehrpersonen, die von der Primarlehrpersonenausbildung mit der Berechtigung für die gesamte Volksschule abgekoppelt wurde, oder die Verlängerung der Ausbildungszeit auf Sek-II-Niveau von vier auf fünf Jahre.

«Hinter den Kulissen» fand im Gefolge der Diskussion um die praktischen Folgerungen aus dem LEMO-Bericht in den frühen 1980er-Jahren eine Auseinandersetzung hinsichtlich der Bewahrung oder Veränderung der Ausbildungsstrukturen für Lehrpersonen der Sek I statt. Dem war eine Auseinandersetzung um die Bewahrung oder Veränderung des konstruierten Bildes von Schülerinnen und Schülern vorgängig. Die tradierte - orthodoxe - Auffassung ging weiterhin von der Annahme klar unterscheidbarer Schülertypen aus, die eine Schultypengliederung mit schultypenorientierten Lehrpersonenausbildungsmodellen plausibel erscheinen liess. Zwar konnte die AGS I die Annahmen der tradierten Auffassung unterscheidbarer Schülertypen mit dem Verweis auf empirische Studien hinterfragen und ein neues sowohl konsequent akademisches als auch stufenorientiertes Sek-I-Lehramt in Vorschlag bringen, scheiterte aber an den Beharrungskräften der Vernehmlassungspartner, die das heteronome Feld der Meinungsbildung zu Ausbildungsmodellen für Lehrpersonen der Sek I bis zur Gründung der pädagogischen Hochschulen beherrschten. Insgesamt zeigte sich das Verhältnis zwischen wissenschaftlicher Expertise und bildungspolitischer Empfehlung als komplexes Problem, da einerseits Gutachten vorlagen, die aus den Erkenntnissen der Pädagogischen Psychologie eine schultypenorientierte Lehrpersonenausbildung «ableiten» wollten, andererseits auch wissenschaftliche Stellungnahmen der empirischen Pädagogik verfügbar waren, die auf eine Sachangemessenheit der stufenorientierten Lehrerinnen- und Lehrerausbildung deuteten. Bourdieu zufolge konkurrenzierten sich in einem Kräftefeld Akteure mit der gleichen Sorte reinen wissenschaftlichen Kapitals (Bourdieu, 1998). Somit führte der Gebrauch bildungswissenschaftlicher Befunde in den 1980erJahren nicht dazu, eine politische Mehrheit für die Einleitung eines Systemwechsels zu schaffen. Wissenschaftliche Bezugnahmen alleine veränderten das Feld der Auseinandersetzung zur Sek-I-Ausbildung nicht. Erst wenn Akteure der Bildungsadministrationen und der Lehrerverbände sich zugunsten einer wissenschaftlichen Position aussprechen, wird eine grundlegende Veränderung von Ausbildungsmodellen wahrscheinlicher.

\section{Anmerkung}

1 Ich danke Hans Ambühl (EDK) für den Zugang zum Archiv der EDK. 


\section{Quellen}

Staatsarchiv Luzern (StA LU):

StA LU, EDK, Akten und Druckschriften 1895-2000, A 1270/835; StA LU, EDK, Akten und Druckschriften 1895-2000, A 1270/836; StA LU, EDK, Akten und Druckschriften 1895-2000, A 1270/837

Erziehungsrat des Kantons Luzern (1984). Bericht «Die Ausbildung der Lehrer für die Sekundarstufe I». StA LU, A 1270/836; A 1270/837

\section{Bibliographie}

Archiv für das schweizerische Unterrichtswesen. (1965/66). Schulen in der Schweiz. I. Institutionen des Bundes. II. Beschreibung nach Kantonen. Frauenfeld: Huber.

Badertscher, H. (1983). Modellvorschläge für die Verbesserung und Koordination der Lehrerbildung für die Sekundarstufe I in der Schweiz. BzL, I (2), 21-23.

Bourdieu, P. (1998). Vom Gebrauch der Wissenschaft. Für eine klinische Soziologie des wissenschaftlichen Feldes. Konstanz: UVK.

Bourdieu, P. (2001). Das politische Feld. Zur Kritik der politischen Vernunft. Konstanz: UVK.

Bucher, Th. (1970). Vorwort. In H. Gehrig (Hrsg.), Die pädagogischen Disziplinen in der Lehrerbildung. Pädagogik. Psychologie. Didaktik (S. 7-8). Basel: Beltz.

Caruso, M. (2011). Bildungspolitik zwischen Expertenwissen und Öffentlichkeit. ZfPäd, 57 (4), 453-458.

Caselmann, Chr. (1964). Wesensformen des Lehrers. Versuch einer Typenlehre. Stuttgart: Klett.

COHEP. (2009). Konsekutiver Masterstudiengang vom Bachelor VS/PS zum Master Sek. I: Antrag der Kommission Ausbildung. Beschlussnummer VS 786. 49/2009, 13.05.2009. Bern: Typoskript.

Criblez, L. (2008). Vom strikten Bildungsföderalismus zur bescheidenen Schulkoordination. In L. Criblez (Hrsg.), Bildungsraum Schweiz. Historische Entwicklungen und aktuelle Herausforderungen (S. 251-276). Bern: Haupt.

Criblez, L. (2010). Die Reform der Lehrerinnen- und Lehrerbildung in der Schweiz seit 1990: Reformprozesse, erste Bilanz und Desiderata. In H. Ambühl \& W. Stadelmann (Hrsg.), Tertiarisierung der Lehrerinnen- und Lehrerbildung (EDK-Schriftenreihe Studien + Berichte) (S. 22-58). Bern: o.V.

Criblez, L. \& Bosche, A. (2007). Die Entwicklung der Sekundarstufe I im Kanton Zürich. PH Akzente, 4, 32-35.

Denzler, St. \& Wolter, St. C. (2008). Selbstselektion bei der Wahl eines Lehramtsstudiums:

Zum Zusammenspiel individueller und institutioneller Faktoren. Beiträge zur Hochschulforschung, 30 (4), 112-141.

Duncker, L. (2002). Schulpraxis «im Lichte von Theorien». Die Bedeutung von Theorie und Praxis in der Professionalisierung des Lehrberufs. Die Deutsche Schule, 94 (1), 21-38.

EDK. (1979). Die Ausbildung der Lehrer der Sekundarstufen I und II. Informationsbulletin 15. Genf: EDK.

EDK. (2005). 4.1.1. Interkantonale Vereinbarung über die Anerkennung von Ausbildungsabschlüssen vom 18. Februar 1993 (Änderung vom 16. Juni 2005). Bern: EDK.

EDK. (2009). 4.2.2.4. Reglement über die Anerkennung von Hochschuldiplomen für Lehrkräfte der Sekundarstufe I vom 26. August 1999. (Totalrevision des EDK-Statuts vom 3. März 2005; Änderung vom 29./30. Oktober 2009). Bern: EDK.

EDK. (2010). 4.2.2.4.1. Richtlinien für die Anerkennung einer Ausbildung zur Lehrperson der Sekundarstufe I auf Masterstufe für Lehrpersonen der Vorschul- und Primarstufe sowie der Primarstufe vom 28. Oktober 2010. Bern: EDK.

Egger, E. (Hrsg.). (1983). Die Ausbildung der Lehrer für die Sekundarstufe I. Bern: Haupt.

Frey, K. et al. (1969). Die Lehrerbildung in der Schweiz. Eine analytische Darstellung der 52 Lehrerbildungsanstalten in Hinsicht auf ihre Reform. Band I der Strukturanalyse der Volks- 
schullehrerbildung. Weinheim: Beltz.

Gehrig, H. (1999). Lehrerbildung im Wandel. Aufsätze und Reden. Zürich: Pestalozzianum.

Gensicke, D. (2006). Irritationen pädagogischer Professionalität. Vermittlungshandeln im Erziehungssystem in Zeiten individualistischer Habitusformen. Heidelberg: Auer.

Gerber, R. (1987). Lehrer im Spannungsfeld von Innovationen. Eine Untersuchung zur Veränderung der Berufssituation durch Reformen am Beispiel des AVO-Modells. Dissertation, Universität Bern.

Grunder, H.-U. (1993). Seminarreform und Reformpädagogik. Bern: Peter Lang.

Haeberlin, U. (Hrsg.). (1972a). Schweizer Gesamtschulmodelle. Eine Zusammenstellung von Berichten über neue Volksschuloberstufenmodelle mit gesamtschulartigen Merkmalen. Basel: Beltz.

Haeberlin, U. (Hrsg.). (1972b). Gesamtschule Schweiz. Materialien zur pädagogischen und schulbaulichen Differenzierung. Zürich: Benziger \& Sauerländer.

Haefeli, H., Schäder, R. \& Häfeli, K. (1979). Schulische Auslese bei Abschluss der Primarschule. Bern: Haupt.

Halbherr, P. (1980). Leistungsnormen im Übergangsbereich Schule/Beruf. Eine empirische Untersuchung an den Oberstufen der Gemeinden Sissach und Allschwil (BL). Bern: Lang.

Herrlitz, H. G., Hopf, W., Titze, H. \& Cloer, E. (2005). Deutsche Schulgeschichte von 1800 bis zur Gegenwart. Weinheim: Juventa.

Herzog, W. (2011). Professionalität im Beruf von Lehrerinnen und Lehrern. In H. Berner \& R. Isler (Hrsg.), Lehrer-Identität. Lehrer-Rolle. Lehrer-Handeln. Professionswissen für Lehrerinnen und Lehrer (S. 49-77). Baltmannsweiler: Schneider.

Hoffmann-Ocon, A. \& Metz, P. (2010). Situierung der Lehrerbildung. In U. Klingovsky, P. Kossack \& D. Wrana (Hrsg.), Die Sorge um das Lernen (S. 237-249). Bern: hep.

Hügli, A. (1989). Die Basler Lehrerbildung und das Problem der Einheit des Lehrerberufs. BzL, 7 (3), 349-354.

Jenzer, C. (1983). Gesamtschule Dulliken 1970-1980: Idee, Realisierung, Resultate, Ausblick. Die Schlussbilanz zum einzigen (öffentlichen) Gesamtschulversuch der deutschen Schweiz. Bern: Haupt.

Jenzer, C. (1998). Schulstrukturen als historisch gewachsenes Produkt bildungspolitischer Vorstellungen. Blitzlichter in die Entstehung der schweizerischen Schulstrukturen. Bern: Peter Lang.

Künzle, P. (2011, 1. März). Znüni-Täschli statt Bildungsrucksack. Politiker und Fachleute kritisieren die Ausbildung der Sekundarlehrer. Basler Zeitung, S. 3.

Künzli, R. (1993). Die Behauptung der Schule durch die Universität. BzL, 11 (1), 63-73.

Laur, Fr. (2011, 1. März). Studienleiter kontert Schelte. Marktwirtschaft und Lehrermangel führen zu Sachzwängen. Basler Zeitung, S. 3.

Messmer, R. (1999). Orte und Nicht-Orte der Lehrerbildung. Eine historische und empirische Untersuchung zur Handlungs- und Wissensorientierung und der damit verbundenen Mythen in der Lehrerbildung. Bern: Peter Lang.

Mietzel, G. (1967). Die Einstellung der Abiturienten zum Volksschullehrerberuf. Eine sozialpsychologische Untersuchung. Braunschweig: Westermann.

Müller, F., Gehrig, H., Jenzer, L., Kaiser, L. \& Strittmatter, A. (Hrsg.). (1975). LEMO-Bericht. Grundlagen. Strukturen. Inhalte. Bericht der Expertenkommission "Lehrerbildung von morgen" im Auftrag der Schweizerischen Konferenz der kantonalen Erziehungsdirektoren. Hitzkirch: Comenius.

Oelkers, J. (1992). Universität und Lehrerbildung. BzL, 10 (2), 185-196.

Oertel, L. (1981). Probleme der Oberstufenreform - Historische Aspekte der Volksschulentwicklung im Kanton Zürich. Bildungsforschung und Bildungspraxis, 3 (3), 251-262.

PAEK. (1993). Sekundarstufe I. Bericht mit Vorschlägen zuhanden der Erziehungs- und Kulturdirektion des Kantons Basel-Landschaft. Arbeitsgruppe Sekundarstufe I. Liestal.

Raschke, J. \& Tils, R. (2007). Politische Strategie. Eine Grundlegung. Wiesbaden: VS-Verlag. Rickenbacher, I. (1975). Erwartungen an den Lehrer von morgen - Folgerungen für die 
Ausbildung. In U. Isenegger, I. Rickenbacher \& A. Strittmatter, Reformbedingungen der Lehrerbildung in der Schweiz (S. 41-54). Basel: Beltz.

Rosenmund, M. (2011). Bildungsförderalismus in der globalisierten Bildungsinstitution -

Das Schulsystem der Schweiz. In K. Kansteiner-Schänzlin (Hrsg.), Schule im gesellschaft-

lichen Spannungsfeld (S. 33-46). Schneider: Baltmannsweiler.

SKBF. (2010). Bildungsbericht 2010. Aarau: SKBF.

Wirz, B. (1993). Zum Abschluss der Arbeiten am NW EDK-Vorprojekt Lehrerinnen- und

Lehrerbildung für die Sekundarstufe I. BzL, 11 (2), 205-209.

Schlagworte: Ausbildungsmodelle Sekundarstufe I, Akademisierung der Lehrerbildung, Reformen der Lehrerbildung in der Schweiz

\section{Les modèles de formation à l'enseignement au degré secondaire I en Suisse alémanique - fondements et conflits}

\section{Ré sumé}

Basé sur les archives de la Conférence suisse des directeurs de l'Instruction publique (CDIP), cet article retrace les tentatives de réformes de la formation des enseignants du secondaire obligatoire qui ont eu lieu durant la deuxième partie du 20 e siècle en Suisse alémanique. Les enjeux liés à l'académisation de la formation et à son orientation vers des niveaux scolaires particuliers ont généré un débat auquel se sont retrouvées confrontées les structures traditionnelles de formation à l'enseignement de chaque canton. La reconstitution historique de ce processus montre que tant les initiateurs des réformes que ceux qui les critiquent tentent de légitimer leur position en invoquant des expertises scientifiques. À partir d'une analyse approfondie des documents produits, l'article démontre que l'argument scientifique seul n'a jamais suffi à provoquer une réforme dans la formation des enseignants.

Mots-clés : Dispositifs de formation à l'enseignement secondaire, académisation et réformes de formation à l'enseignement secondaire. 


\section{Modelli di formazione per I docenti del secondario I nella Svizzera tedesca: linee fondamentali e conflitti.}

\section{Riassunto}

A partire dagli archivi della CDPE, questo articolo si occupa dei tentativi di riforma della formazione degli insegnanti per il settore secondario I e del Volksschuloberstufe nella seconda metà del XX secolo nella Svizzera tedesca. I temi dell accademizzazione e dell orientamento al settore scolastico hanno generato una linea di conflitto che ha irritato le strutture tradizionali delle formazioni cantonali. Attraverso una ricostruzione di storia della foramzione si mostra che sia I promotori che I detrattori delle riforme hanno cercato legittimare le loro argomentazioni con argomenti scientifici. Sulla base di un analisi documentale larticolo arriva alla conclusione che un rargomentazione scientifica non è sufficiente, da sola, e provocare una trasformazione nella formazione degli insegnanti.

Parole chiave: Dispositivi di formazione all'insegnamento secondario, accademizzazione e riforme dell'insegnamento secondario

\section{Common and embattled grounds: Models of teacher education for secondary level I in German-speaking Switzerland}

\section{Summary}

Based on historical sources from the Swiss Conference of Cantonal Ministers of Eduction, the paper deals with attempts of teacher training reforms for secondary schools in the second half of the 20th century in German-speaking Switzerland. Academisation and school level orientation formed a cleavage, which irritated the traditional structures of teacher training in every canton. Educational historical reconstruction reveals that both, the initiators of the reform and its critics were trying to support their positions with scientific expertise. Drawing upon a document analysis, this article argues that scientific reference alone has never been sufficient to trigger a regime change in teacher training.

Keywords: Models of teacher education for secondary level I in Germanspeaking Switzerland, academisation of teacher education. 
